\title{
Development of strategy for ensuring operability of transport and technological machines
}

\author{
Sergey Repin ${ }^{1, *}$, Sergey Maximov ${ }^{1}$, Andrei Zazykin ${ }^{1}$, and Nikolay Voropaev ${ }^{2}$ \\ ${ }^{1}$ Saint Petersburg State University of Architecture and Civil Engineering, 190005, St. Petersburg, \\ Russia \\ ${ }^{2}$ St. Petersburg University of the State Fire Service of the Ministry of Emergencies of Russia, \\ 196105, St. Petersburg, Russia
}

\begin{abstract}
The article presents the results of research in the field of ensuring the performance of complex technical objects on the example of transport and technological machines (TTM), which include transport equipment, as well as technological machines (excavators, loaders and other equipment that performs a technological process). The strategy of forming a system of maintenance and repairs taking into account the technical condition of TTM elements is described. The strategy provides for the division of structural elements of machines into four groups, depending on the principle approach to calculating the frequency of their repair. The criterion for determining the frequency of repairs is the probability of reaching the maximum values of characteristics during the inter-repair period, as well as the degree of criticality of the consequences of possible failure of the element.
\end{abstract}

\section{Introduction}

The efficiency of transport and technological machines (TTMs), as well as of any material object, is determined by the level of their reliability, which is defined as a property to maintain for a set period of time a certain range of the values of all parameters that characterize the ability to function in the set modes and application conditions [GOST 27.002-83]. Reliability is a comprehensive property that includes fail-free operation, longevity, maintainability, and storability. The term "operability" is of similar meaning. It is defined as the condition of an object upon which it is capable of its intended functions while maintaining the values of operating (set) parameters within the range established by the regulatory documents. The parameters, that characterize functioning, determine the performance figures of a machine, i.e. its performance efficiency, load capacity, power capacity, propulsion performance, speed performance, and braking performance, motor burn process parameters, etc. From the consumer's point of view, the term "operability" is more understandable, with its parameters being obvious and measurable.

\footnotetext{
* Corresponding author: repinserge@mail.ru
} 
Loss of operability, i.e. the state of non-operability, occurs due to a failure. A failure can be either sudden (machine breakdown) or parametric (contingency). Operability of complex equipment, which includes TTMs, is ensured through maintenance and repair (M\&R). The main purpose of M\&R is to reduce the probability of sudden failures to a reasonable level, i.e. to ensure the basic property of reliability - failure-free operation, and to maintain the values of parameters within the permissible limits. The solution is to perform timely maintenance or replacement of the machine components that affect its operability.

Repair is conducted in accordance with the current advanced operation strategy condition-based repair. In terms of probability of sudden failures, the condition evaluation is carried out based on statistic operational data for this component, and the repair time is set based on the chosen probability of failure-free operation. In terms of parametric operability, the condition evaluation is carried out based on the parameters of the assemblies under repair, determined through diagnostics. Repair (replacement) of components is carried out before the limiting values of probability of failure-free operation or values of the parameters are reached.

The limiting values of the reliability indicators and the parameters of components' condition are chosen with consideration for the type of an object, nature of its application, structural diagram of its reliability, the type (or the way of its determining) of the limit state. For example, the type of an object is a vehicle. Let us consider two types of vehicles, a passenger plane and an agricultural tractor. Failure of an important component in a plane during a flight will result in a plane crash, while such failure in a tractor will lead to a stop in operation in a field. Consequences of failures are very different, which is why the types of the limit state also differ. The limit state for a plane is the value of the probability of failure during a flight, determined by the safety requirements. For a tractor, the value of the probability of failure is determined by the requirements of the economic feasibility of its use (Bujaczek, 2013, Kotikov, 2017 et al.). The structural diagram is defined by the availability of element redundancy. In a plane, the majority of the systems are redundant, while in a tractor, only the brake system is redundant.

The dynamics of the current values of the components' condition parameters during operation is monitored through regular diagnostics according to the M\&R schedule or through continuous diagnostics by means of special instrumentation embedded into the operated object, and by means of observations by personnel. Dynamics of the current values of the reliability indicators is evaluated based on the collected statistical data on the components' failures.

Maintenance, repair, and component replacement are scheduled with consideration for the peculiarities of the object's operation. For example, for transport construction machines (pipelines) used seasonally in places which are less accessible for M\&R, the time between repairs will be long. That is why the term of the service life of the machine components before repair or replacement is increased respectively. This results in the increase in the scope of repair and replacement works before the season when the machine is in operation. Such condition-based M\&R method can be called a preventive one.

\section{Materials and methods}

The subject of the research is a TTM as a complex technical system that consists of components, the frequency of replacement of which determines the reliability of the whole machine.

The objective of the article is to develop methods for the justification of the assembly replacement frequency.

The methods of statistical analysis and prediction have been used. 
The structural diagram of ensuring condition-based operability of TTMs is presented in Fig.1. The condition-based operation strategy is carried out in the following way (Trukhanov, 2014).

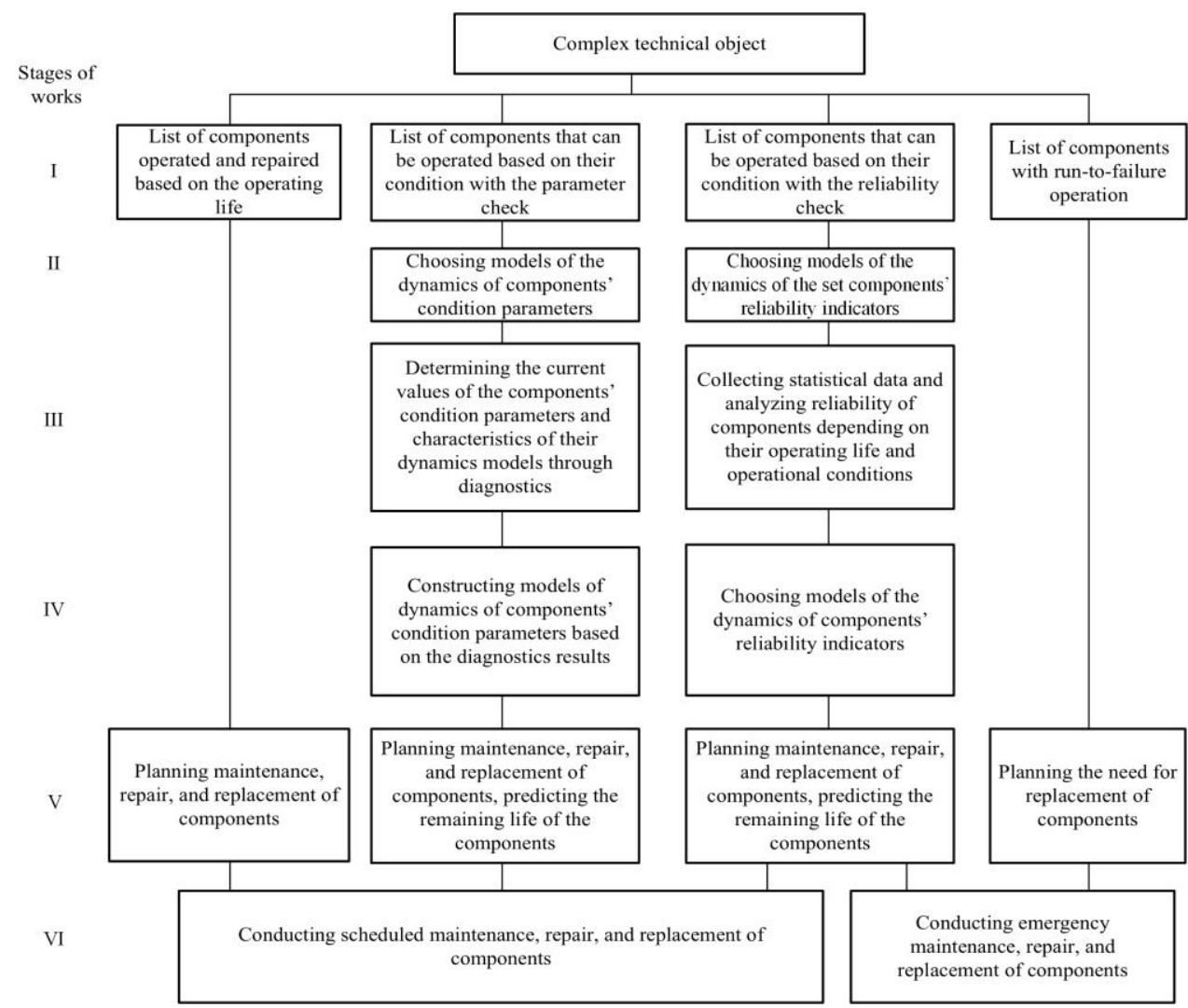

Fig. 1. Structural diagram of ensuring condition-based operability of a complex technical object.

Stage 1 - operating object structure analysis. The list of operating components with reliability check, condition parameters check, based on operating life and run-to-failure is composed.

Stage 2 - constructing mathematical models of dynamics of the components' condition indicators in order to predict them and determine the schedule of their preventive repair and replacement.

The form of mathematical models, limiting values of the reliability indicators and the parameters of components' condition are chosen with consideration for the type of an object, nature of its application, structural diagram of its reliability, the type (or the way of its determining) of the limit state.

The dynamics of the current values of the components' condition parameters during operation is monitored through regular diagnostics according to the M\&R schedule or through continuous diagnostics by means of special instrumentation embedded into the operated object, and by means of observations by personnel (Stage 3). Dynamics of the current values of the reliability indicators is evaluated based on the collected statistical data on the components' failures.

Through the substitution of the current values of reliability indicators and components' condition parameters into the mathematical dynamics models, prediction of the term of preventive maintenance and replacement (Stage 4) is carried out. Based on this term, the 
M\&R activities and replacement of components are scheduled (Stage 5). Maintenance, repair, and component replacement are scheduled with consideration for the peculiarities of the object's operation.

According to the developed schedules, scheduled maintenance, repair and replacement of components are conducted. Emergency repairs are carried out after a sudden failure of a machine's component. The number of sudden failures is predicted based on the statistical data on failures of either machines of this make or machines of other makes that are similar to that machine with regard to their parameters (assemblies by the same manufacturers, design concepts, conditions of usage, etc.). Reserve repair capacity and spare parts are provided based on the prediction of sudden failures.

\section{Results and discussion}

\subsection{Composing lists of components operated and repaired using various strategies}

The list of components to be repaired based on the operating life is composed according to the regulation recommended by the manufacturer. This group of components includes drive belts of control shafts, brake shoes, filters, and other high-wear parts, rubber products, and engineering maintenance materials. A delay in replacement of such components may result in the machine breakdown.

The condition parameters are monitored in components when changes in the condition parameters can be measured through diagnostics, and the time of pre-failure condition can be determined (Makhutov, et al., 2013). Those are the components that become unserviceable primarily due to parametric failures.

Components that are operated based on the reliability level are the components that become unserviceable primarily due to sudden failures.

The components with run-to-failure operation are the components, failure of which does not result in severe consequences or does not significantly affect the operability of the machine. Those are illumination devices, finishing details, protective covers, safety components, etc.

The frequency of component repair and replacement conducted as per regulations, if such components had run-to-failure operation, is determined conclusively, and planning of such measures is easy. However, planning of condition-based repair requires special research conducted for specific types of machines and operation conditions.

\subsection{Planning repair and replacement of machine components based on the condition parameters' dynamics}

The values of $X i$ characteristics of parameters of the component condition are measured by means of diagnostics when conducting scheduled M\&R or through continuous built-in diagnostics (Fig. 2). 


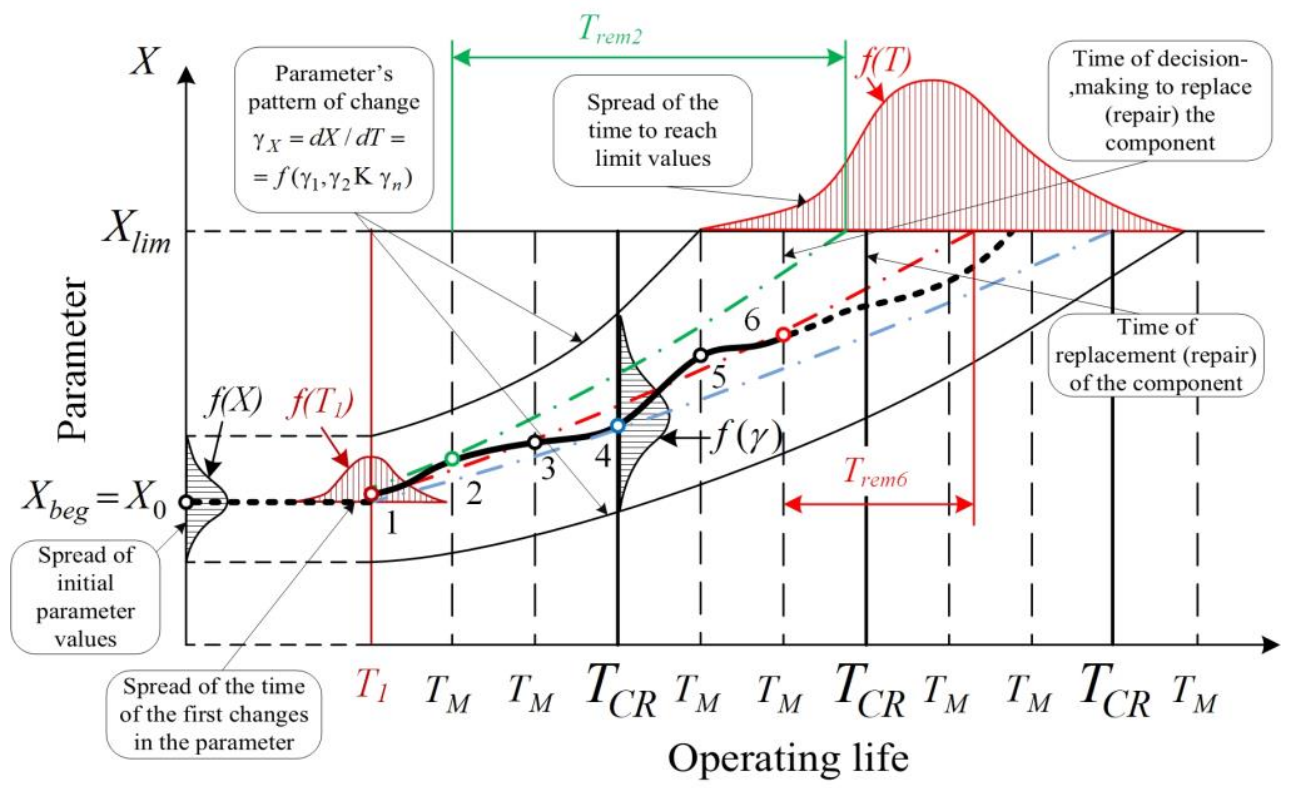

Fig. 2. Curve of the pattern of the component condition with condition parameters' monitoring: $1 \ldots 6$ - $X$ parameter sensing points; $T_{M}, T_{C R}$ - frequency of maintenance and current repair, respectively; $T_{\text {rem } 2}, T_{\text {rem } 6}-$ remaining service life of a component at the $2^{\text {nd }}$ and $6^{\text {th }}$ measurements of $X$ parameter, respectively.

Remaining service life $T_{\text {remi }}$ is calculated by the measured value of $X_{i}$ parameter and the known pattern of its change $X(T)$. This remaining service life determines the calculated time to the limiting state of the component, which is characterized by the $X_{\text {lim }}$ value of the parameter. Based on $T_{\text {remi }}$, the decision on further operation of the component or its repair is made. Fig. 3 shows that repair of the component shall be conducted during the second current repair. Thus, the main task is to determine the remaining service life of the component based on each measurement of the parameter.

The limiting state may be set based on economic parameters (Repin, 2015), reliability, or other criteria. For example, for excavating machines operating as a part of a technological chain of machines at road construction, the limiting state is the reduction in the discharge of the pump by $5 \%$, since it will result in corresponding reduction in the performance of the whole production process. And for excavating machines operating at emergency repair of underground pipework, reduction in the discharge of the pump by $20 \%$ is admissible, since excavation makes up such a small portion of the total repair time that its performance will have virtually no effect on the time of repair.

Let us consider a general pattern of parametric failure occurrence when various damage processes result in the time changes of result parameter X. A failure occurs when the parameter reaches its admissible limit value $X_{\text {lim }}$, which will occur in a certain random period of operation of the item.

The chart shows the main stages of the $f(T)$ distribution law. Initially, dispersion of component $f(T)$ parameters relative to its statistical expectation $X_{0}$ takes place. This is associated with the dispersion of the initial performance of a new machine. The changes in the parameters of components during operation of the machines are affected by slow processes, for example, wear. Generally, the process of the parameter change can start in a certain period of time $T_{1}$, which is also a random value and is associated with the damage accumulation (for example, fatigue damage) or external reasons. 
The process of the changes in $X$ parameter with the rate of $\gamma \mathrm{x}$ is also random and depends on the changes in the damage of individual elements of the component (their wear with the rate of $\gamma_{1}, \gamma_{2}, \ldots \gamma_{\mathrm{n}}$ ) (Pavlov, 2012).

As a result of these phenomena, distribution law $f(X, T)$ is formed. It determines the probability of parameter $\mathrm{X}$ exceeding the limiting value $X_{\text {lim }}$. It should be noted that, generally, $X_{\lim }$ value can also have dispersion, provided that it assesses the range of the consumer's requirements to the limiting values of the machine parameters.

This chart describes a general process of formation of a gradual (parametric) failure, and, in case of particular values of input parameters, it can reflect certain cases typical for certain operational conditions and design features of an item. If the process of the parameter changes starts immediately $\left(T_{l}=0\right)$, the formation of a gradual parametric failure is standard. If $X(T)$ abruptly increases when $X_{\text {lim }}$ is reached, then a functional failure usually occurs. If, in the process of formation of a failure, the primary case is the occurrence (onset) of the process, i.e. function $f\left(T_{l}\right)$, and then the process is very intensive $(X(T) \rightarrow \infty)$, it means the sudden failure model.

Dispersion of initial parameters of component $f(X)$ shall be taken into account when considering a certain item population, for example, all machines of this make manufactured by a certain plant. When an individual item is considered, value $\mathrm{X}$ becomes non-random, since it characterizes initial parameters of this sample.

On the contrary, when we consider dispersion of initial parameters of a machine after its operation in different modes, $\mathrm{X}$ will be a random value for this sample.

Typically, changes in item parameter $\mathrm{X}$ are dependent of the linear law

$$
X(T)=X_{O}+k \cdot T .
$$

In this case, $k=\gamma$ is the rate of the process (the rate of wear or the rate of the parameter changes), which usually depends on a large number of random factors, such as load, rate, temperature, operating conditions, etc.

In a general case, the pattern of function $k(T)$ or $\gamma(T)$ may vary. This pattern may be known initially or determined during the analysis of operational data.

Fig. 1 shows that the pattern of $X_{i}$ parameter changes, measured in i points, is variable. That is why rate $\gamma$ of the parameter changes is also variable.

$$
\gamma_{i, i-1}=\frac{X_{i}-X_{i-1}}{T_{i}-T_{i-1}}
$$

where $X_{i}$ is a measured value of the parameter at moment $T_{i}$. Average rate of the parameter changes.

$$
\gamma_{\text {medium }}=\frac{\sum_{i=2}^{n} \gamma_{i, i-1}}{n-1}
$$

where $n$ is the number of measurements of the parameter.

The case when the spread of the parameter changes values is dependent on the normal law is the most typical:

$$
f(\gamma)=\frac{1}{\sigma \sqrt{2 \pi}} \exp \left(-\frac{(\gamma-\bar{\gamma})^{2}}{2 \sigma^{2}}\right)
$$


where $f(\gamma)$ is the probability density; $\bar{\gamma}$ is the statistical expectation; $\sigma$ is the mean root square deviation of the process rate.

The admissible limit value of parameters $X_{\text {lim }}$ is determined based on the condition of correct operation of the item. When $X=X_{\text {lim }}$, the limit state is reached. It determines the service (operating) life of the item before failure $T=T_{f}$.

Average lifetime of an item:

$$
T_{\mathrm{av}}=\left(X_{\mathrm{lim}}-X_{0}\right) / \gamma_{\mathrm{av}} .
$$

The remaining service life determined based on the results of the measurements of the parameter value in $i$ point.

$$
T_{\mathrm{rem} i}=\left(X_{\mathrm{lim}}-X_{i}\right) / \gamma_{\mathrm{av}}
$$

If the resultant value of the remaining service life is less than the frequency of M\&R, the component shall be repaired during the nearest current maintenance.

\subsection{Planning repair and replacement of machine components based on the component reliability level}

Any machine is a complex technical system reliability of which is determined by its components (Makhutov, Reznikov, 2015; Shao-Fei et al., 2014; Protasov, Nikolaychuk, 2011). When analyzing the structural reliability of a system, we can determine the reliability indicators of its components (Chernyavsky, Shadchin, 2010). The reliability of machine components is the function of their operating life. When setting a certain level of components' reliability, and being familiar with the time law of reliability, we can figure out the frequency of replacement of the components.

The reliability of a component can be determined based on various criteria (Trukhanov, 2008). Let us consider a probabilistic model for determining the time before preventive replacement based on the criterion of minimizing the weighted-average costs for repairs. The model has been proposed by professor E. I. Zaytsev (Trukhanov, 2008). As the weight coefficients for two options (emergency repair caused by a sudden failure, and preventive repair), there have been used, respectively, the risk of missing a failure (corresponds to $\left.F\left(T_{r}\right)\right)$

$$
R_{\mathrm{r}}=\int_{0}^{T r} f(T) d T=F\left(T_{\mathrm{r}}\right)=1-P\left(T_{\mathrm{r}}\right)
$$

and the risk of overspending on preventing failures (corresponds to the probability of failure-free operation).

$$
R_{\mathrm{p}}=\int_{T r}^{0} f(T) d T=P\left(T_{\mathrm{r}}\right)
$$

where $f(T)$ is the probability density for the time before failure with statistical expectation $\bar{T}_{f} ; T_{r}$ is the time to preventive repair.

The costs to be reduced are determined by the formula:

$$
Z=Z_{\mathrm{r}} \cdot R_{\mathrm{r}}+Z_{\mathrm{p}} \cdot R_{\mathrm{p}}=Z_{\mathrm{p}}\left[1-P\left(T_{\mathrm{r}}\right)\right]+Z_{\mathrm{p}} \cdot P\left(T_{\mathrm{r}}\right) \rightarrow \min ,
$$


where $Z_{r}$ is the total cost of emergency repair; $Z_{p}$ is the total cost of preventive repair.

If the components of the cost are expressed in terms of the average cost of emergency repair $C_{\mathrm{r}}$ (taking into account damage $\mathrm{D}$, both direct and collateral damage caused by the hold-up) and preventive repair $C_{p}$, then:

$$
Z=T_{o v}\left\{\left(C_{\mathrm{p}}+Y\right) \cdot\left[1-P\left(T_{\mathrm{r}}\right)\right]+Z_{\mathrm{p}} \cdot P\left(T_{\mathrm{r}}\right)\right\} \cdot T_{\mathrm{r}}^{-1} \rightarrow \min ,
$$

where $T_{o v} / T_{r}$ is the number of operating (repair) cycles with the set ultimate operating life $T_{o v}$ (for example, before overhaul - OV).

The Mathcad code has been developed for this example. In a graphical form, Fig. 3 shows the results of the calculations of the most preferable periods for replacement of components with minimum cost for the repair cycle for various values of $\mathrm{D}$ (damage).

The calculations have been made for two kinds of replaced components of shovel excavators (SEs) - high-pressure hoses (HPHs) and dipper teeth. The average time before failures $\left(\bar{T}_{f}\right)$ of an HPH is commensurate to the overhaul time (6500 and 8000 running hours, respectively), which is why the number of replacements between overhauls does not exceed two, which reduces the demonstrational value of some diagrams. However, the damage related to a sudden failure (D is up to 10,000 RUB) affects HPHs significantly, since in case of HPH rupture, a large number of expensive power fluid is drained away. For dipper teeth, $\bar{T}_{f}=400 \ldots 500$ running hours, which is why the number of replacements for components with different reliability indicators varies significantly. Fig. 3 shows the impact, the reliability characteristics of the components replaced have on the value of the most preferable periods of component replacement, calculated taking into account the minimum expenditures for the repair cycle. Using this analytical method allows treating the optimization of spare parts procurement in terms of price/quality ratio (Matvienko, 2014).

The impact, the amount of damage from sudden failures of an equipment component has on the most preferable period of its replacement, is shown in Fig. 5. The calculations have been performed for two replaced components with various reliability characteristics' parameters.

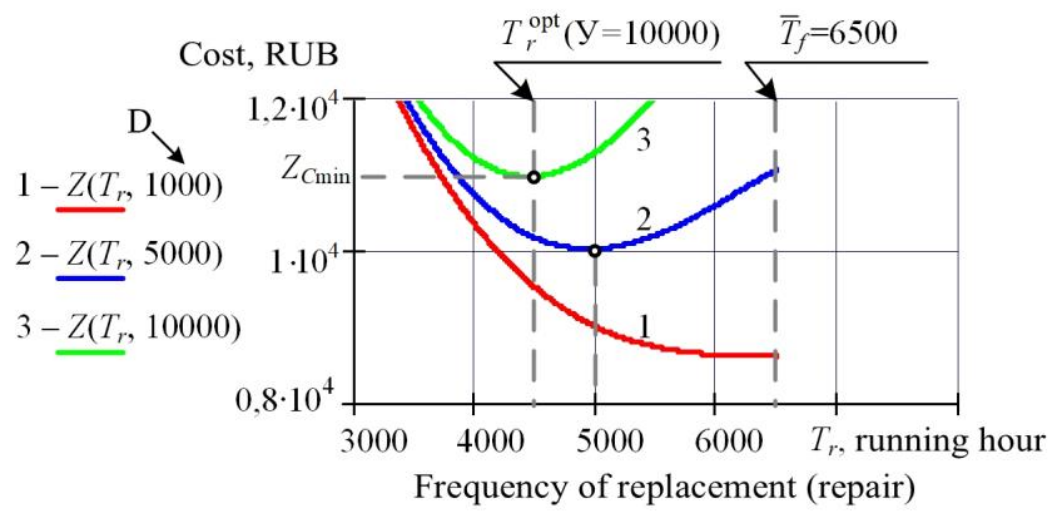

Fig. 3. Results of the calculations of the most preferable periods of replacement of components (HPHs) with minimum cost for the repair cycle for various values of damage D (in RUB): $1-1000$, $2-5000,3-10000$. 


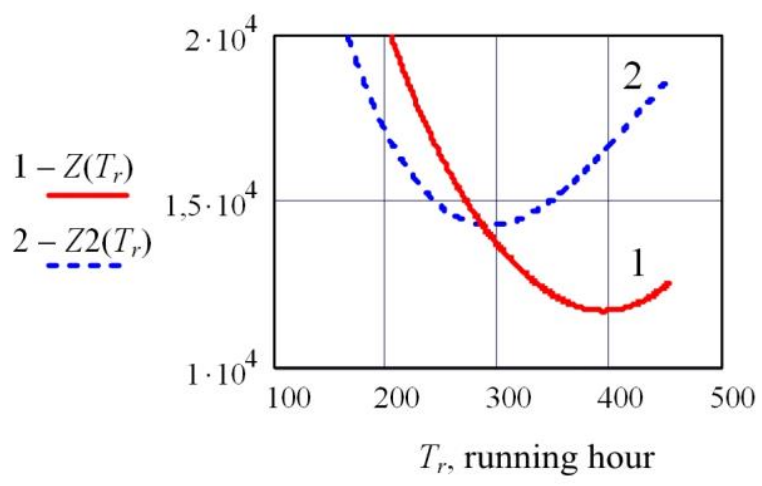

Fig. 4. Impact of the reliability of replaced components (dipper teeth) on the most preferable periods of component replacement, calculated taking into account the minimum cost of the repair cycle (see characteristics in Table 1).

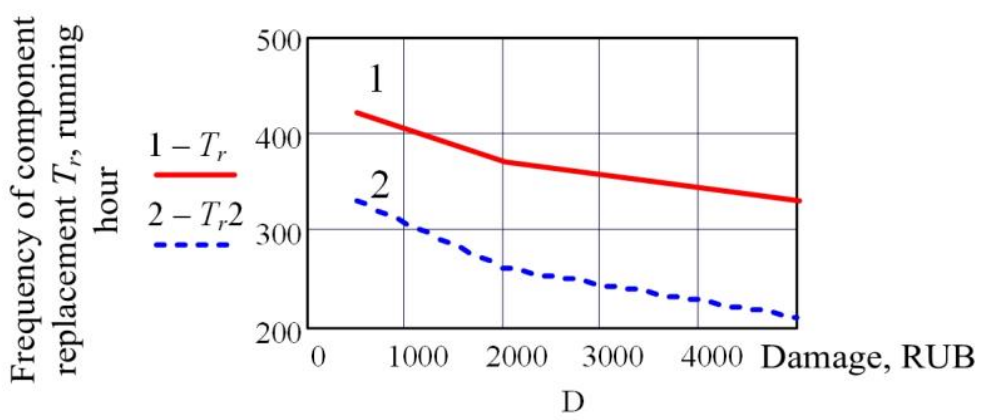

Fig. 5. Impact of the amount of damage from sudden failures of an equipment component (a tooth) and its characteristics on the most preferable periods of replacement (as exemplified by a dipper tooth).

The value of $T_{r}$ after the optimization is the basis for the calculation of the need for spare parts, and it is reasonable to coordinate replacement with the nearest (the prior one is preferable) scheduled M\&R.

The method of calculating the frequency and the number of replacements of an equipment component also makes it possible to solve the task in terms of ensuring the set probability of failure-free operation. Fig. 5 shows the chart and the results of such calculations in a graphical form. Fig. 7 shows the results of the calculations for components with various reliability levels. 


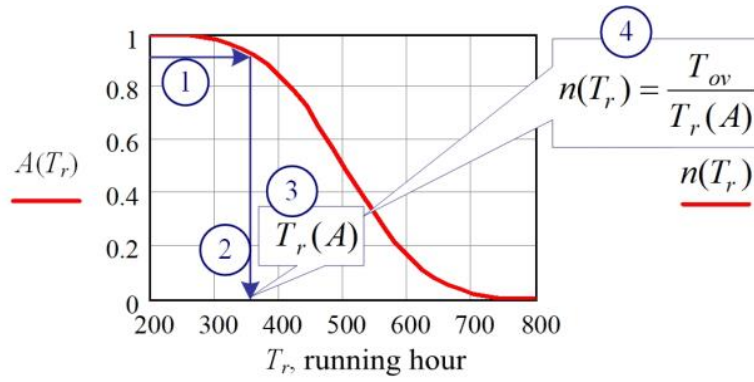

a

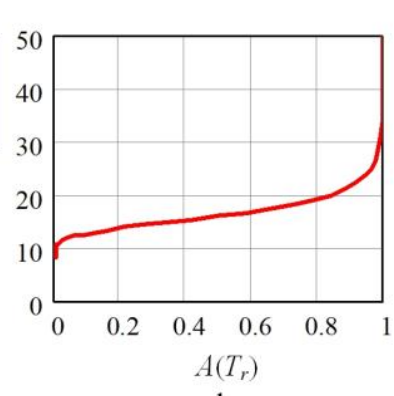

$\mathrm{b}$

Fig. 6 Determining the number of replacements $\mathrm{n}\left(T_{r}\right)$ of an equipment component depending on the required probability of failure-free operation: where $a$ is the relation of the probability of failure-free operation $A\left(T_{r}\right)$ with frequency $T_{r}$ of replacements of an equipment component (running hours); $b$ is the dependence of the number of replacements $n\left(T_{r}\right)$ of an equipment component on the required probability of its failure-free operation $A\left(T_{r}\right) ; T_{o v}$ is the scheduled overhaul time.
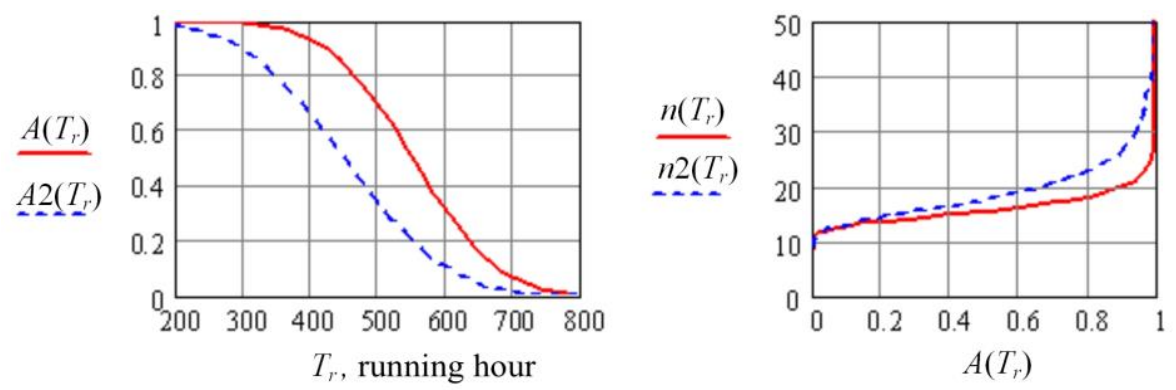

Fig. 7. Impact of the reliability of a replaced component (a dipper tooth) on the frequency (a) and the number (b) of its replacements.

\section{Conclusion}

The described strategy of establishing a system of maintenance and repair for transport and technological machines allows setting the time of repair and replacement of their components based on the results of the analysis of their condition, as well as on the set values of the limit state indicators and reliability.

The impact of the amount of possible damage from a sudden failure on choosing the frequency of repairs of machine components has been described.

The research has been conducted with financial support from the Russian Humanities Research Foundation as part of the R\&D project Development of the method to establish structural elements of the transport system based on economic and reliability criteria (as exemplified by the transport and technological machine fleets), Project No. 15-02-00512.

\section{References}

1. R. Bujaczek, K. Sławińskii, A. Grieger, J. Techn. Sc., 16, 1, 13-18 (2013)

2. A. Chernyavsky, A. J. Mach. Manuf. Rel., 39, 4, 402-406 (2010)

3. J. Kotikov, J. Arch. Eng., 2, 3, 21-25 (2017)

4. N. Makhutov, A. Fomin, V. Ivanov, V. Permyakov, I. Vasil’ev, J. Mach. Man. Rel., 42, 2, 109-113 (2013) 
5. N. Makhutov, D. Reznikov, J. Mach. Man. Rel., 44, 8, 675-686 (2015)

6. G. Matvienko, J. Mach. Man. Rel., 43, 3, 242-249 (2014)

7. V. Pavlov, J. Mach. Man. Rel., 41, 5, 431-434 (2012)

8. A. Protasov, J. Mach. Man. Rel., 40, 1, 27-30 (2011)

9. S. Repin, J. Appl. Mech. Mat., 725-726, 990-995 (2015)

10. S. Repin, K. Rulis, A. Zazykin, S. SPBGASU (2012)

11. J. Shao-Fei, Fu Da-Bao, Wu Si-Yao, J. Math. Probl. Eng. (2014)

12. M. Trukhanov, J. Mach. Man. Rel., 37, 5, 530-533 (2008)

13. V. Trukhanov, J. Mach. Man. Rel., 43, 2, 124-126 (2014) 